\title{
Reconstrucción de Imágenes Optoacústicas: Efecto de los Sensores Piezoeléctricos de Banda Ancha
}

\author{
P. Massaro Rocca*, L. Ciocci Brazzano*†, E. Acosta*, M. G. González ${ }^{* \dagger 1}$ \\ *Universidad de Buenos Aires, Facultad de Ingeniería, \\ Grupo de Láser, Óptica de Materiales y Aplicaciones Electromagnéticas (GLOMAE) \\ Paseo Colón 850, C1063ACV, Buenos Aires, Argentina \\ ${ }^{\dagger}$ Consejo Nacional de Investigaciones Científicas y Técnicas, (CONICET) \\ Godoy Cruz 2290, C1425FQB, Buenos Aires, Argentina \\ ${ }^{1}$ mggonzalfi.uba.ar \\ Recibido: 25/09/17; Aceptado: 13/11/17
}

\begin{abstract}
Optoacoustic imaging is a hybrid technique with a rapid evolution within the area of biomedical imaging. It combines the rich and versatile optical contrast with the high spatial resolution associated with the low-dispersion ultrasonic wave propagation nature. Its application raises a series of difficult problems that require further developments. One of them, the objective of this work, is to analyze how the optoacoustic image reconstruction algorithms are affected by the use of non-ideal (real) broadband sensors. In particular, as an original contribution, we study the case of integrating ultrasonic sensors based on piezoelectric polymer thin films. In order to achieve this, a temporary back-projection algorithm was implemented for line detectors. Then, using a parametric model for this type of sensors, we studied the influence of the use of a real device on the efficiency of the implemented algorithm.
\end{abstract}

Resumen- La obtención de imágenes optoacústicas es una técnica híbrida con una rápida evolución dentro del área de imágenes biomédicas. Ésta se beneficia tanto del rico y versátil contraste óptico como de la alta resolución espacial asociada con la naturaleza de baja dispersión de propagación de las ondas ultrasónicas. Su aplicación plantea una serie de difíciles problemas que exigen ulteriores desarrollos. Uno de ellos, el objetivo de este trabajo, es analizar cómo afecta a los algoritmos de reconstrucción de imágenes optoacústicas el uso de sensores no ideales (reales) de banda ancha. En particular, como aporte original, se estudia el caso de los sensores ultrasónicos extensos basados en películas delgadas de polímeros piezoeléctricos. Para lograr esto, se implementó un algoritmo de retroproyección temporal para detectores lineales. Luego, usando un modelo paramétrico para este tipo de sensores, se estudió la influencia del uso de un dispositivo real sobre la eficiencia del susodicho algoritmo.

\section{INTRODUCCIÓN}

La tecnología para la obtención de imágenes optoacústicas (IOA) de origen biológico está actualmente en pleno desarrollo, generando nuevos enfoques técnicos y aplicaciones. En comparación con la formación de imágenes ecográficas por ultrasonido (IEU), las amplitudes de las señales OA son relativamente bajas pero su contenido espectral es amplio, abarcando frecuencias desde varias decenas de $\mathrm{kHz}$ hasta un centenar de $\mathrm{MHz}$ para estructuras a escala micrométrica. De hecho, se ha demostrado que es posible generar IOA a partir de tejido vivo con una resolución espacial del orden de los micrones, lo que permite la obtención de información bioquímica relevante [1].

Una configuración típica OA consta de tres elementos esenciales: una fuente de luz, un sistema de detección de las ondas acústicas y uno para el procesamiento de las señales. Por ejemplo, en el modo de excitación pulsada, el tejido es iluminado por un láser que emite pulsos de luz monocromática con una duración típica de algunos nanosegundos. Las tasas de repetición de pulsos son del orden de unas pocas decenas de Hertz, con energías en el rango de microJoules por pulso. Cuando esta técnica es utilizada para realizar tomografía (TOA), los perfiles de presión generados por la excitación óptica son captados con sensores que rodean la zona de interés. Por lo tanto, se puede decir que la tecnología de formación de IOA es híbrida, lo que le permite beneficiarse tanto del rico y versátil contraste óptico como de la alta resolución espacial (limitada por difracción) asociada con la naturaleza de baja dispersión de propagación de las ondas ultrasónicas, en comparación con las electromagnáticas [2].

Es posible clasificar los sensores ultrasónicos usados en la obtención de IOA en dos categorías: transductores piezoeléctricos, en los cuales la presión medida es directamente proporcional a la señal eléctrica, y detectores ópticos, los cuales son sensibles a cambios en el largo del camino óptico inducido por ondas de presión [3]. Los transductores piezoeléctricos son los más comúnmente utilizados y están basados en materiales poliméricos (sensores de banda ancha) o cerámicos (sensores resonantes). Desde el punto de vista geométrico, estos sensores son de dos tipos: de pequeña apertura (puntuales) o de gran apertura (extensos). En los de pequeña apertura los efectos de difracción deben ser compensados por los algoritmos de reconstrucción de la imagen y, por otro lado, tienen la ventaja de que es posible hacer un arreglo con múltiples elementos. De esta forma se lleva a cabo una rápida adquisición de imágenes con resolución adecuada. En el caso de un detector de gran apertura se puede conseguir una imagen de alta resolución angular y, si el detector tiene un gran ancho de banda, se tiene una buena discriminación en distancias [4].

Para conseguir imágenes a partir de señales OA se deben resolver dos problemas inversos: uno acústico y otro óptico [5]. En ambos casos se parte de las señales acústicas medidas. En el problema inverso acústico se intenta mapear la energía depositada en la muestra, mientras que el objetivo del problema inverso óptico es conseguir la imagen del coeficiente de absorción. La TOA aplicada a la obtención 
de imágenes de objetos vivos es la aplicación que mayores desafíos presenta para resolver ambos problemas inversos. Ópticamente, las grandes variaciones en los coeficientes de dispersión y absorción de los tejidos vivos lleva a problemas inversos no lineales muy complejos. Por otro lado, acústicamente, la geometría del sistema de detección, así como también la heterogeneidad y pérdidas usualmente presentes en la muestra, conducen a distorsiones y artefactos en las imágenes obtenidas [5].

Existe una gran cantidad de técnicas de inversión para obtención de imágenes en TOA que pueden ser agrupadas dentro de 4 categorías: I) algoritmos en el dominio del tiempo (retroproyección); II) algoritmos en el dominio de la frecuencia; III) algoritmos de inversión del tiempo y IV) aquellos basados en modelos matriciales [5]. Las primeras dos categorías, en gran medida, permiten obtener soluciones cerradas al problema de inversión. El enfoque que mejores resultados ha tenido en los trabajos experimentales es el algoritmo de retroproyección, el cual es muy simple de implementar y es aceptable para la mayoría de las configuraciones experimentales para obtención de IOA, aún cuando no es exacta. Por este motivo es que en este trabajo nos abocaremos a este enfoque.

Los algoritmos en el dominio del tiempo se basan en proyectar cada una de la señales temporales OA unidimensionales en el espacio tridimensional de una forma que es consistente con el principio de tiempo de vuelo [5]. El proceso de retroproyección generalmente involucra tres pasos. El primer paso es el pre-procesamiento sobre cada una de la señales acústicas medidas, cuya operación depende de la implementación particular del algoritmo. Luego se realiza la retroproyección, la cual consiste en tomar cada una de las señales pre-procesadas y proyectarlas sobre esferas concéntricas en el espacio 3D. Finalmente, para obtener la imagen, se realiza la suma sobre todos los datos calculados en el paso anterior.

En la Fig.1 se puede observar un esquema del proceso de retroproyección 2D. Una señal acústica originada en la posición $\mathbf{r}$ es captada por 4 sensores (Fig. 1.a). Las señales medidas poseen un retardo proporcional al módulo de la distancia entre la ubicación de la fuente y el sensor (Fig. 1.b). Cada una de las señales se proyecta en arcos de circunferencia con un radio igual a la distancia mencionada. La única posición en donde todos los arcos se intersectan y donde la contribución de todas las señales retroproyectadas se suman coherentemente es en la posición $\mathbf{r}$. Si la fórmula de reconstrucción es exacta y el número de sensores es suficientemente grande, se puede recuperar el punto fuente.

Existen muchas implementaciones de este enfoque, siendo la más completa el algoritmo de retroproyección universal desarrollado por $\mathrm{M}$. Xu y coautores [6] que entrega una solución exacta para las superficies de detección más comunes: esférica, cilíndrica y plana.

Los algoritmos de reconstrucción de imagen, generalmente, suponen sensores ideales. En este trabajo se define a un sensor ideal como el caso límite donde su señal de salida sigue instantáneamente a la presión acústica. Entre los polímeros que se usan para estos sensores, se puede encontrar el fluoruro de polivinilideno (PVDF) y sus copolímeros, que presentan gran interés debido a sus propiedades físicas


Fig. 1. Ilustración del proceso de retroproyección 2D. (a) Un punto fuente es captado por 4 sensores. (b) Señales temporales medidas por los sensores. (c) Cada una de las señales se proyecta en arcos de circunferencia con un radio igual a la distancia entre el punto fuente y el sensor.

que permiten su utilización en muchas aplicaciones. Estos materiales son flexibles, están disponibles como películas delgadas, tienen un gran ancho de banda acústica, y sus valores de impedancia acústica están próximos a los del agua y los tejidos biológicos (a las frecuencias de interés) [7]. Las características del PVDF y sus copolímeros han sido extensamente estudiadas, destacándose que sus ventajas son relevantes para transductores de recepción de ultrasonido [8].

Sensores del tipo descripto distan de ser ideales. Dentro de nuestro conocimiento no hay trabajos que estudien detalladamente las consecuencias del uso de sensores polímericos piezoeléctricos no ideales en los algoritmos de reconstrucción de imagen.

En este trabajo nos enfocaremos en los aspectos técnicos del problema inverso acústico en TOA, estudiando cómo las distorsiones introducidas por el sensor afectan a los algoritmos para reconstrucción de las imágenes. En particular, se analiza la influencia del uso de sensores poliméricos piezoeléctricos de gran apertura sobre la eficiencia del algoritmo de retroproyección universal que se describe en la siguiente sección.

\section{Algoritmo DE RETROPROYECCIÓN UNIVERSAL}

Cuando la duración del pulso láser es mucho más corta que los tiempos de relajación térmica y mecánica involucrados en el proceso OA, la presión en una posición $\mathbf{r}=$ $(x, y, z)$ responde a la ecuación de onda [9],

$$
\left(\frac{\partial^{2}}{\partial t^{2}}-c^{2} \nabla^{2}\right) p(\mathbf{r}, t)=0
$$

con las siguientes condiciones iniciales, 


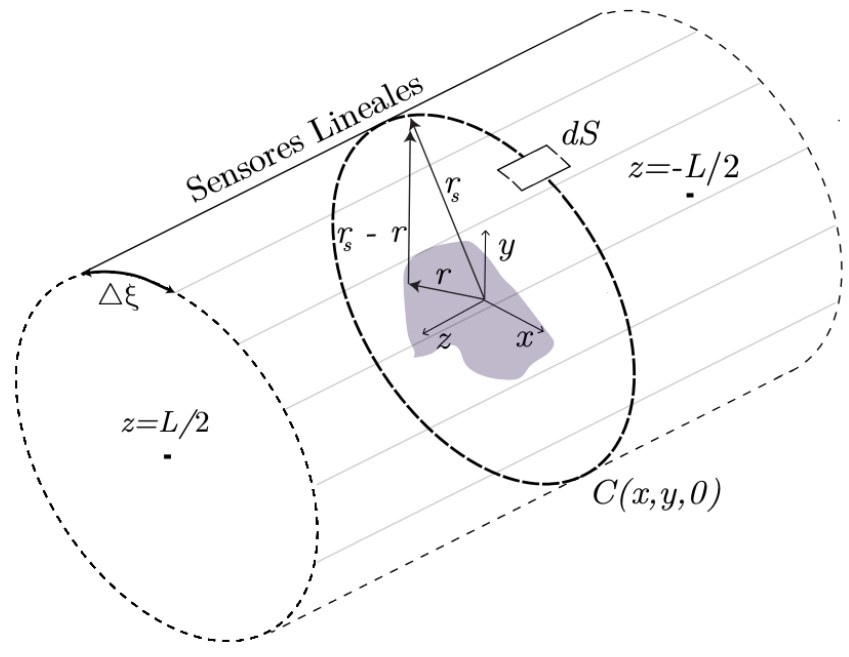

Fig. 2. Esquema para obtención de IOA 2-D con sensores lineales.

$$
p(\mathbf{r}, 0)=p_{0}(\mathbf{r}) \quad\left(\frac{\partial p}{d t}\right)(\mathbf{r}, 0)=0 \quad \mathbf{r} \in \mathbb{R}^{3}
$$

donde $c$ es la velocidad del sonido en el medio y $p_{0}(\mathbf{r})$ es el perfil espacial de presión inicial generado por la muestra.

La clave del problema inverso es reconstruir la presión inicial $p_{0}(\mathbf{r})$ partiendo de los datos medidos. La señal OA captada por un sensor puntual en la posición $\mathbf{r}_{s}$ se puede expresar como [4]:

$$
p_{d}\left(\mathbf{r}_{\mathbf{s}}, t\right)=\frac{\partial}{\partial t}\left[\frac{t}{4 \pi} \iint_{\left|\mathbf{r}_{\mathbf{s}}-\mathbf{r}\right|=c t} p_{0}(\mathbf{r}) d \Omega\right]
$$

donde $d \Omega$ es el elemento de ángulo sólido del vector $\mathbf{r}$ respecto de $\mathbf{r}_{\mathbf{s}}$.

Si se ubican sensores puntuales ideales sobre una superficie $S$ plana o cilíndrica alrededor del objeto irradiado, se puede utilizar el algoritmo de retroproyección universal [6] para obtener el perfil inicial de presión:

$$
\begin{gathered}
p_{o}(r)=-\frac{2}{c^{2} \Omega_{S}} \int_{S} f_{u b p}\left(\mathbf{r}_{\mathbf{s}},\left|\mathbf{r}-\mathbf{r}_{\mathbf{s}}\right|\right) \cos (\alpha) d S \\
f_{u b p}=\left(t^{-1} \frac{\partial p}{\partial t}\right)
\end{gathered}
$$

donde $\Omega_{S}$ es $2 \pi$ o $4 \pi$ ya sea una superficie plana o cilíndrica, respectivamente, y $\alpha$ el ángulo entre el vector normal de la superficie de detección $\mathbf{n}_{\mathbf{s}}$ y el vector $\mathbf{r}_{\mathbf{s}}-\mathbf{r}$. $f_{u b p}$ es una función de pre-procesado utilizada para resaltar características de la señal. Si bien existen diversas formas funcionales de $f_{u b p}$, todas ellas cumplen con la finalidad de filtrar las componentes de baja frecuencia y dejar pasar las altas frecuencias, con un comportamiento lineal en el medio [6]. Esto es muy necesario en retroproyección ya que el método en sí mismo realza las componentes de baja frecuencia. Por lo tanto, con $f_{u b p}$, se acentúan las características de contraste de la imagen (altas frecuencias), mientras que se minimiza el efecto de imágenes borrosas (bajas frecuencias).

\section{A. Algoritmo de retroproyección 2-D}

$\mathrm{Si}$ en lugar de detectores puntuales sobre una superficie $S$, se dispusieran sensores lineales a lo largo de una circunferencia $C$ y solidarios con el eje $z$ (ver Fig. 2), la señal de presión captada por uno de estos sensores ubicado en la posición $\left(x_{s}, y_{s}, z\right)$ es:

$$
p_{s}\left(x_{s}, y_{s}, t\right)=\int_{-L / 2}^{+L / 2} p\left(x_{s}, y_{s}, z, t\right) d z
$$

Bajo la suposición de que la distribución inicial de presión tiene soporte acotado, es decir, $\partial p(r) / \partial z=0 \mathrm{y}$ $\partial^{2} p(r) / \partial z^{2}=0$ para $|z| \geq L / 2$, se puede establecer que:

$\int_{-\infty}^{+\infty} \frac{\partial^{2} p(\mathbf{r})}{\partial^{2} z} d z=\int_{-L / 2}^{L / 2} \frac{\partial^{2} p(\mathbf{r})}{\partial^{2} z} d z=\left[\frac{\partial p(\mathbf{r})}{\partial z} d z\right]_{z=-L / 2}^{z=L / 2}=0$

Utilizando la igualdad anterior, se puede probar que $p_{s}\left(x_{s}, y_{s}, t\right)$ satisface la ec. (1) bidimensional:

$$
\begin{gathered}
\left(\frac{\partial^{2}}{\partial t^{2}}-c^{2} \frac{\partial^{2}}{\partial x^{2}}-c^{2} \frac{\partial^{2}}{\partial y^{2}}\right) p_{s}\left(x_{s}, y_{s}, t\right)=0 \\
p_{s}\left(x_{s}, y_{s}, 0\right)=p_{s_{0}}\left(x_{s}, y_{s}\right)=\int_{-\infty}^{+\infty} p_{0}\left(x_{s}, y_{s}, z\right) d z \\
\frac{\partial p_{s}}{\partial t}\left(x_{s}, y_{s}, 0\right)=0
\end{gathered}
$$

Por lo tanto, a partir de las señales captadas por detectores lineales dispuestos sobre la curva $C$, es posible reconstruir la proyección 2-D de la distribución de presión inicial en el plano $x y$. Este método está descripto en el trabajo de $\mathrm{P}$. Burgholzer, et al. [9]. A continuación se detallan los pasos para adaptar la ec. (3) al esquema planteado en la Fig. 2.

Integrando a lo largo del eje $z$ a ambos lados de la ec. (3):

$p_{0}(x, y)=-\frac{2}{c^{2} \Omega_{S}} \int_{-\infty}^{\infty}\left[\int_{S} f_{u b p}\left(\mathbf{r}_{\mathbf{s}},\left|\mathbf{r}-\mathbf{r}_{\mathbf{s}}\right|\right) \cos (\alpha) d S\right]_{(7)} d z$

siendo ahora $\cos (\alpha)$ definido como:

$$
\cos (\alpha)=\mathbf{n}_{\mathbf{s}} \cdot \frac{\mathbf{r}_{\mathbf{s}}-\mathbf{r}}{\left|\mathbf{r}_{\mathbf{s}}-\mathbf{r}\right|}=\cos (\gamma) \frac{\rho}{\sqrt{\rho^{2}+\left(z-z_{s}\right)^{2}}}
$$

donde $\gamma$ es el ángulo entre el vector normal a la curva $C$, $\mathbf{n}_{\mathbf{c}}, \mathbf{y}\left(x_{s}-x, y_{s}-y\right)$, y $\rho=\left[\left(x-x_{s}\right)^{2}+\left(y-y_{s}\right)^{2}\right]^{1 / 2}$ es la distancia entre el punto $(x, y)$ y la ubicación del sensor $\left(x_{s}, y_{s}\right)$.

Cambiando el orden de integración de la ec. (7) y reescribiendo el diferencial de superficie, $p_{o}(x, y)$ es igual a:

$$
\begin{gathered}
\frac{-2}{\Omega_{S} c^{2}} \int_{C}\left(\int_{-\infty}^{\infty} \int_{-\infty}^{\infty} \frac{f_{u b p}\left(\mathbf{r}_{\mathbf{s}}, \beta\right)}{\beta} d z d z_{s}\right) \rho \cos (\gamma) d C \\
\beta=\sqrt{\rho^{2}+\left(z-z_{s}\right)^{2}}
\end{gathered}
$$

Teniendo en cuenta la simetría de la disposición de los sensores respecto del eje $z$ y sustituyendo $z-z_{s}=\bar{z}$, la 
integral interna de la ec. (9) puede evaluarse a través de la siguiente expresión:

$$
2 \int_{0}^{\infty} f_{u b p}\left(x_{s}, y_{s}, \sqrt{\rho^{2}+\bar{z}^{2}}\right) d \bar{z}
$$

Si la velocidad del sonido $c$ en el medio es constante y homogénea, se puede establecer una relación sencilla entre el módulo de la distancia y el tiempo. De esta forma, se realiza un cambio de variables integrando en $t$ en lugar de $\bar{z}$ obteniendo:

$$
p_{0}(x, y)=\frac{-4}{c^{2} \Omega_{S}} \int_{C}\left(\int_{\rho}^{\infty} \frac{f_{u b p}\left(x_{s}, y_{s}, t\right)}{\sqrt{t^{2}-\rho^{2} / c^{2}}} d t\right) w(\rho, \gamma) d C
$$

donde $w=\rho \cos (\gamma)$ y se denomina factor de peso.

Cabe destacar que el método detallado entrega una solución exacta si la superficie de detección $S=C \mathrm{x} \mathbb{R}$ es un cilindro o un plano infinito. Por lo tanto, la contraparte bidimensional será exacta si $C$ es una circunferencia o una línea infinita.

\section{B. Discretización del algoritmo}

La ec. (11) requiere un conjunto continuo de datos. Dado que en una aplicación práctica esto no es posible, resulta necesario discretizarla.

En primer lugar se parametriza la curva $C$ como $\left(x_{s}, y_{s}\right)=\left(x_{s}(\xi), y_{s}(\xi)\right)$, utilizando un parámetro $\xi$ que pertenece a un intervalo $I$. Entonces la ec. (11) puede reescribirse de la siguiente forma:

$$
\begin{gathered}
\frac{4}{c^{2} \Omega_{S}} \int_{I}\left(\int_{\rho / c}^{\infty} \frac{f_{u b p}\left(x_{s}, y_{s}, t\right)}{\sqrt{t^{2}-\rho^{2} / c^{2}}} d t\right) w(\rho, \gamma) h_{C} \\
h_{C}=\sqrt{\left(\frac{d x_{s}}{d \xi}\right)^{2}+\left(\frac{d y_{s}}{d \xi}\right)^{2}} d \xi
\end{gathered}
$$

Luego, se realiza un muestreo uniforme temporal y espacial del eje de tiempo y de la curva $C$, respectivamente,

$$
\begin{aligned}
t^{m} & =m \Delta t & & m \in\left[0, \ldots, N_{t}-1\right] \\
\xi^{k} & =k \Delta \xi & & k \in\left[0, \ldots, N_{\xi}-1\right]
\end{aligned}
$$

donde $\Delta t$ y $\Delta \xi$ son los pasos de tiempo y de longitud (curva $C$ ), respectivamente. $N_{t}$ representa la cantidad de muestras del eje de tiempo y $N_{\xi}$ la cantidad de sensores empleados. Por lo tanto, la presión del sensor $k$ para el tiempo discreto $t^{m}$ es: $p^{m, k}=p\left(x_{s}\left(\xi^{k}\right), y_{s}\left(\xi^{k}\right), t^{m}\right)$.

El siguiente paso es discretizar la integral interna de la ec. (12). La función $f_{u b p}$ es aproximada usando el método de diferencias finitas de segundo orden:

$$
q^{m, k}=\left(\frac{p^{m+1, k}}{t^{m+1}}-\frac{p^{m-1, k}}{t^{m-1}}\right) /\left(2 c^{2} \Delta t\right)
$$

con $q^{0, k}=0$.

Para evaluar la función en puntos no muestreados, se realiza una interpolación lineal en el intervalo,

$$
L^{k}[q](t)=q^{m, k}+\frac{t-t^{m}}{\Delta t}\left(q^{m+1, k}-q^{m, k}\right)
$$

donde $t \in\left[t^{m}, t^{m+1}\right]$

Luego, la integral interna de la ec. (12) es evaluada para $\rho / c=t_{m}$ y la función de pre-procesado es reemplazada por su versión discreta e interpolada:

$$
\begin{gathered}
\hat{q}^{m, k}:=\int_{t^{m}}^{\infty} \frac{L^{k}[q]}{\sqrt{t^{2}-\left(t^{m}\right)^{2}}} d t= \\
\sum_{m^{\prime}=m}^{N_{t}-1} q^{m^{\prime}, k}\left(\int_{t^{m^{\prime}}}^{t^{m^{\prime}+1}} \frac{d t}{\sqrt{t^{2}-\left(t^{m}\right)^{2}}}\right)+ \\
+\sum_{m^{\prime}=m}^{N_{t}-1} \frac{q^{m^{\prime}+1, k}-q^{m^{\prime}, k}}{\Delta t}\left(\int_{t^{m^{\prime}}}^{t^{m^{\prime}+1}} \frac{\left(t-t^{m^{\prime}}\right) d t}{\sqrt{t^{2}-\left(t^{m}\right)^{2}}}\right)
\end{gathered}
$$

Para que la implementación numérica sea eficiente y precisa, es crucial que las integrales de la ec. (15) sean evaluadas de forma analítica:

$$
\begin{gathered}
\hat{q}^{m, k}=\sum_{m^{\prime}=m}^{N_{t}-1} a_{m^{\prime}}^{m} q^{m^{\prime}, k}+\frac{1}{h_{r}} \sum_{m^{\prime}=m}^{N_{t}-1} b_{m^{\prime}}^{m}\left(q^{m^{\prime}+1, k}-q^{m^{\prime}, k}\right) \\
a_{m^{\prime}}^{m}:=\left[\log \left(t+\sqrt{t^{2}-\left(t^{m}\right)^{2}}\right)\right]_{t=t^{m^{\prime}}}^{t^{m^{\prime}+1}} \\
b_{m^{\prime}}^{m}:=t^{m^{\prime}} a_{m^{\prime}}^{m}+\left[\sqrt{t^{2}-\left(t^{m}\right)^{2}}\right]_{t=t^{m^{\prime}}}^{t^{m^{\prime}+1}}
\end{gathered}
$$

Para evaluar la función $\hat{q}$ en puntos no muestreados, se realiza también una interpolación lineal como el de la ec. (14).

El último paso consiste en evaluar la integral de línea correspondiente a la curva $C$ de forma numérica:

$$
\overline{p_{0}}\left(x^{\mathbf{i}}, y^{\mathbf{i}}\right) \cong p_{0}^{\mathbf{i}}=-\frac{4}{c^{2} \Omega_{S}} \sum_{k=0}^{N_{\xi}} L^{k}[\hat{q}]\left(\rho^{k, \mathbf{i}}\right) w^{k, \mathbf{i}} h_{C}^{k}
$$

donde $\left(x^{\mathbf{i}}, y^{\mathbf{i}}\right), \mathbf{i} \in[0, \ldots, N]^{2}$, representan los puntos pertenecientes a una red uniforme del área que encierra la curva $C$ y $\rho^{k, \mathbf{i}}=\left[\left(x^{\mathbf{i}}-x_{s}^{k}\right)^{2}+\left(y^{\mathbf{i}}-y_{s}^{k}\right)^{2}\right]^{1 / 2}$ es la distancia entre el punto $\left(x^{\mathbf{i}}, y^{\mathbf{i}}\right)$ y la posición del sensor $\left(x_{s}^{k}, y_{s}^{k}\right)$.

Debido a que las diferencias finitas, la interpolación lineal y el método trapezoidal son aproximaciones de segundo orden, una cota error del algoritmo se puede expresar como [9]:

$$
\left|p_{0}^{\mathbf{i}}-\overline{p_{0}}\left(x^{\mathbf{i}}, y^{\mathbf{i}}\right)\right| \leq \kappa \cdot \max \left\{\Delta t^{2}, \Delta \xi^{2}\right\}+\epsilon\left(N_{t} \Delta t\right)
$$

donde $\kappa$ es un constante independiente de i, $\Delta t$ y $\Delta \xi$ $\epsilon\left(N_{t} \Delta t\right)$ representa el error de truncamiento de la integral interna de la eq. (11) 


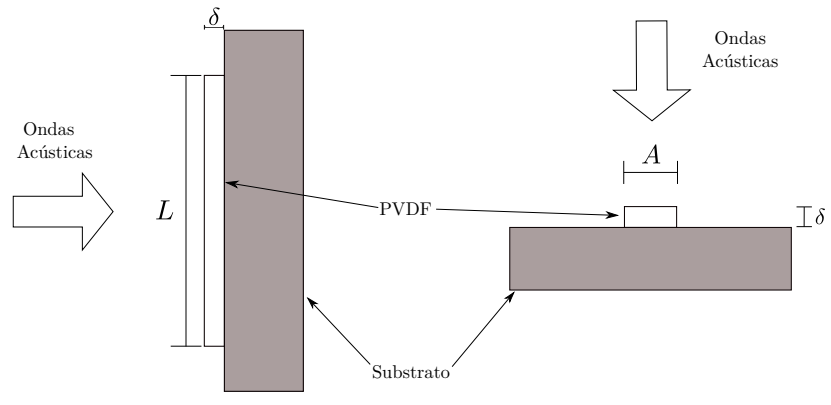

Fig. 3. Esquema (no a escala) del sensor. Vista lateral (izq.) y superior (der.)

\section{MODELO DEL SENSOR POLIMÉRICO PIEZOELÉCTRICO}

Para modelar este tipo de transductores se usa el modelo parámetrico descrito en [10]. Este modelo se caracteriza por: i) suponer que cada punto de la superficie del sensor se comporta como un detector puntual que puede ser aproximado como un sistema lineal e invariante en el tiempo (LTI); ii) los sistemas LTI que componen la superficie son idénticos; iii) las principales características del sensor se modelan a través de funciones transferencia o filtros aplicadas sobre cada uno de los sistemas LTI.

Nuestro estudio se enfoca en sensores de película delgada de PVDF de gran apertura y cuya área de detección puede aproximarse a una línea (cuyo largo $L$ es mucho mayor que su ancho $A$ ). Se denomina cara anterior del film a aquella que se encuentra del lado de donde provienen las ondas acústicas generadas en la muestra. Consecuentemente, la cara opuesta, es la cara posterior (ver Fig. 3).

Es necesario agregar que, dentro de nuestro conocimiento, no hay modelos comerciales de sensores piezoeléctricos con geometría lineal para obtención de IOA. Sin embargo, existen numerosos trabajos de investigación donde han sido aplicados, incluso en sistemas de detección para TOA [11].

En este trabajo consideramos dos aspectos físicos del sensor: las propiedades del material polimérico que lo componen y aquellos relativos con su forma y la relación con su entorno.

Una presión $p_{s}\left(\mathbf{r}_{s}, t\right)$ incidente en la cara anterior del sensor induce un desplazamiento eléctrico $D\left(\mathbf{r}_{s}, t\right)$ en el mismo puede ser calculado a través de la siguiente ecuación [10]:

$$
D\left(\mathbf{r}_{\mathbf{s}}, \omega\right)=P\left(\mathbf{r}_{s}, \omega\right) \cdot d_{33}(\omega)
$$

donde $d_{33}$ es el coeficiente piezoeléctrico y $D\left(\mathbf{r}_{s}, \omega\right)$ y $P\left(\mathbf{r}_{s}, \omega\right)$ las transformadas de Fourier del desplazamiento eléctrico y la presión, respectivamente. En el caso del PVDF, y suponiendo que la onda acústica solo viaja en la dirección perpendicular a su superficie (material de reacción normal) [12], $d_{33}$ puede ser descripto por una función HavriliakNegami [10]:

$$
d_{33}(\omega)=d_{33}^{\infty}+\frac{\Delta d_{33}}{\left(1+\left(i \tau_{0} \omega\right)^{\alpha}\right)^{\beta}}
$$

donde $d_{33}^{\infty}$ es el valor límite del coeficiente piezoeléctrico a altas frecuencias, $\Delta d_{33}$ la intensidad del proceso de relajación, $\tau_{0}$ el tiempo característico de la relajación y $\alpha$ y $\beta$ son los parámetros de forma que describen la asimetría y ensanchamiento de la relajación, respectivamente. Estos parámetros de forma son ambos números positivos y su producto es siempre menor que 1 [13].

Suponiendo que la velocidad de propagación del sonido en el PVDF, $v_{p}$, no depende de la frecuencia, la densidad de carga eléctrica, $\sigma_{p}$, puede ser determinada a partir de la siguiente expresión [10]:

$$
\sigma_{p}\left(\mathbf{r}_{s}, \omega\right)=D\left(\mathbf{r}_{s}, \omega\right) \cdot W\left(\omega, \Gamma_{p}, \Gamma_{a}, \delta, v_{p}, a_{p}\right)
$$

La función $W$ incorpora las reflecciones de las ondas acústicas en las caras posterior y anterior del sensor asî como también el espesor $\delta$ y la atenuación acústica del film piezoeléctrico:

$$
\begin{gathered}
W\left(\omega, \Gamma_{p}, \Gamma_{a}, \delta, v_{p}, a_{p}\right)= \\
\mathcal{F}\left\{\frac { v _ { p } } { \delta } \left\lceil\prod\left(\frac{v_{p} t}{\delta}\right)+\Gamma_{p} \prod\left(\frac{v_{p} t-\delta}{\delta}\right)+\right.\right. \\
\left.\left.+\Gamma_{p} \Gamma_{a} \prod\left(\frac{v_{p} t-2 \delta}{\delta}\right)\right] e^{-a_{p} v_{p} t}\right\}
\end{gathered}
$$

donde $\mathcal{F}$ es el operador transformada de Fourier, $a_{p}$ el factor de atenuación de la onda acústica al propagarse a través del film, $\Pi\left(\frac{z}{\delta / v_{p}}\right)$ una ventana rectangular entre $0 \mathrm{y} \delta / v_{p}, \mathrm{y}$ $\Gamma_{a}$ y $\Gamma_{p}$ los coeficientes de reflexión de la cara anterior y posterior, respectivamente. Esta ecuación supone que $\Gamma_{a}$, $\Gamma_{p}$ y $a_{p}$ son independientes de la frecuencia. Cabe destacar que esta hipótesis fue corroborada experimentalmente en el trabajo [10]. Las sucesivas reflexiones de la onda acústica entre las caras anterior y posterior del film son reemplazadas por el equivalente de propagación de la onda a través de varias capas. En esta ecuación son consideradas solo dos reflexiones ya que se supone que el material polimérico presenta grandes pérdidas.

Finalmente, la carga eléctrica $q(t)$ en los electrodos del film de PVDF se determina a través de la siguiente ecuación:

$$
q(t)=A \int_{-L / 2}^{L / 2} \mathcal{F}^{-1}\left\{\sigma_{p}\left(\mathbf{r}_{s}, w\right)\right\} d z
$$

donde $\mathcal{F}^{-1}$ es la transformada de Fourier inversa. La señal que entrega el sensor al sistema de detección es proporcional a $q(t)$. Es necesario agregar que, este tipo de sensores suele emplearse en conjunto con un amplificador de transimpedancia. En el caso que el ancho de banda del mismo sea mucho mayor que el del sensor, $q(t)$ se puede obtener simplemente integrando la señal medida.

\section{Simulación y Resultados}

En este trabajo se considera que el tiempo característico del pulso láser que irradia a la muestra es mucho menor que cualquiera de los otros tiempos involucrados en el proceso OA. En este caso el objeto dentro de la muestra que absorbe la luz irradiada por el láser es una esfera sumergida en agua a una temperatura $T_{a}=298 \mathrm{~K}$. La esfera tiene un radio $a$ y su centro se ubica en la posición $\mathbf{r}_{a}$. La presión acústica 


\begin{tabular}{|l|l||l|l|}
\hline \multicolumn{2}{|c||}{ Parámetros Fijos } & \multicolumn{2}{c|}{ Parámetros Variables } \\
\hline & valor de referencia & & valor inicial \\
\hline$\delta$ & $25 \mu \mathrm{m}$ & $\Gamma_{a}$ & -0.44 \\
\hline$\tau_{0}$ & $140 \mathrm{~ns}$ & $\Gamma_{p}$ & $-0.99,-0.10$ \\
\hline$\Delta d_{33}$ & $34.5 \mathrm{pC} / \mathrm{N}$ & $a_{p}$ & $2 \cdot 10^{4} \mathrm{~m}^{-1}$ \\
\hline$d_{33}^{\infty}$ & $1.1 \mathrm{pC} / \mathrm{N}$ & $a$ & $10-100 \mu \mathrm{m}$ \\
\hline$v_{p}$ & $2200 \mathrm{~m} / \mathrm{s}$ & $R_{C}$ & $0.1-1 \mathrm{~mm}$ \\
\hline$\alpha$ & 0.5 & $\Delta z$ & $1-10 \mathrm{um}$ \\
\hline$\beta$ & 0.68 & $\Delta t$ & $1 \mathrm{~ns}$ \\
\hline$c$ & $1500 \mathrm{~m} / \mathrm{s}$ & $N_{\xi}$ & 128 \\
\hline$L$ & $20 \mathrm{~mm}$ & $N_{t}$ & 7000 \\
\hline
\end{tabular}

TABLA I

PARÁMETROS FIJOS Y VARIABLES USADOS EN LA SIMULACIÓN. TODOS LOS VALORES SON PARA LA TEMPERATURA DE TRABAJO $T_{a}=298 \mathrm{~K}$.

generada por esta esfera en la posición $\mathbf{r}$ en función del tiempo se calcula a través de la siguiente expresión:

$$
p(\mathbf{r}, t)=\Upsilon \frac{a^{2}-\left(\left|\mathbf{r}-\mathbf{r}_{a}\right|-c t\right)^{2}}{2\left|\mathbf{r}-\mathbf{r}_{a}\right|} H\left(a-|| \mathbf{r}-\mathbf{r}_{a}|-c t|\right)
$$

donde $H$ es la función de Heaviside y $\Upsilon$ una constante de proporcionalidad cuyo valor depende de las propiedades del líquido que rodea a la esfera y de la energía láser absorbida por la misma en el proceso OA.

En todas las simulaciones, con el objetivo de minimizar el efecto de discretización de la curva $C$, se usó un valor de $N_{\xi} \geq 128$. Para calcular la presión captada por el sensor, se discretiza su largo de forma uniforme con un muestreo espacial $\Delta z=0.1 \cdot a$. Por otra parte, la curva $C$, donde se ubican los detectores, es una circunferencia de radio $R_{C}=$ $10 \cdot a$. Estos dos parámetros se eligen en función del tamaño de la fuente esférica para facilitar las comparaciones entre simulaciones. El valor del parámetro $N_{t}$ depende del $\Delta t$ elegido y del tiempo final de integración.

La respuesta de un sensor ideal es:

$$
q(t)=A d_{33}(0) \sum_{k=1}^{L / \Delta z} p\left(\mathbf{r}_{k}, t\right)
$$

Para obtener la respuesta de un sensor no ideal se utilizó el modelo paramétrico detallado en la sección anterior. Se consideraron los siguientes aspectos físicos del sensor : i) su espesor; ii) reflexiones en la cara posterior y anterior (desadaptación de impedancias acústicas); iii) propiedades del material polimérico (pérdidas electromecánicas y relajación piezoeléctrica). Para mostrar el efecto de la desadaptación de impedancias en la cara posterior sobre la reconstrucción de la imagen, se hicieron un conjunto de simulaciones para dos casos de interés experimental: $\Gamma_{p}=-0.99$ (aire) y $\Gamma_{p}=-0.10$ (acrílico).

Los valores de los distintos parámetros utilizados en las simulaciones se muestran en la tabla I. Los valores de referencia relacionados con el PVDF fueron obtenidos de [14].

La integral de la ec. 22 se calcula numéricamente usando la función quadv disponible en GNU/octave.

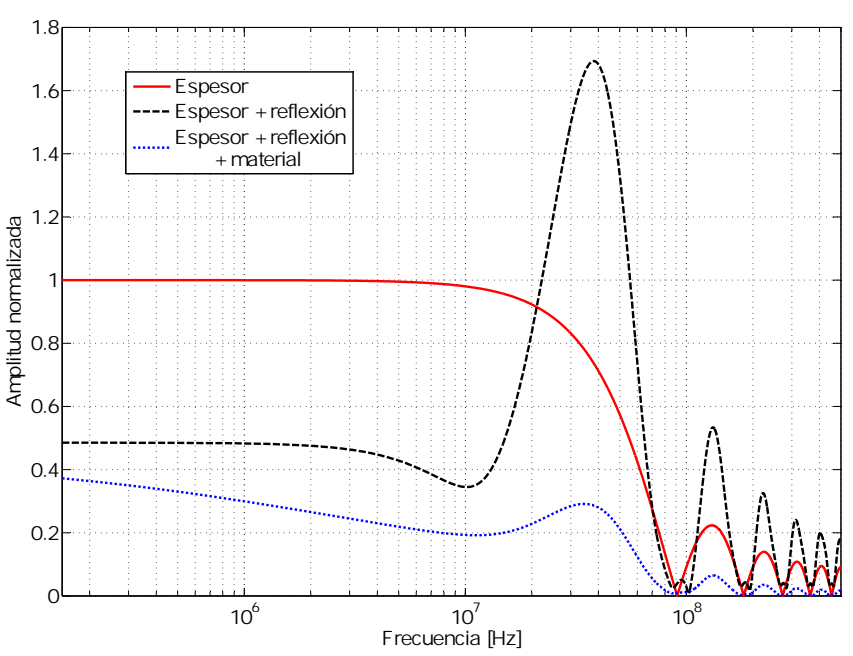

Fig. 4. Módulo normalizado de las transferencias, con $\Gamma_{p}=-0.99$.

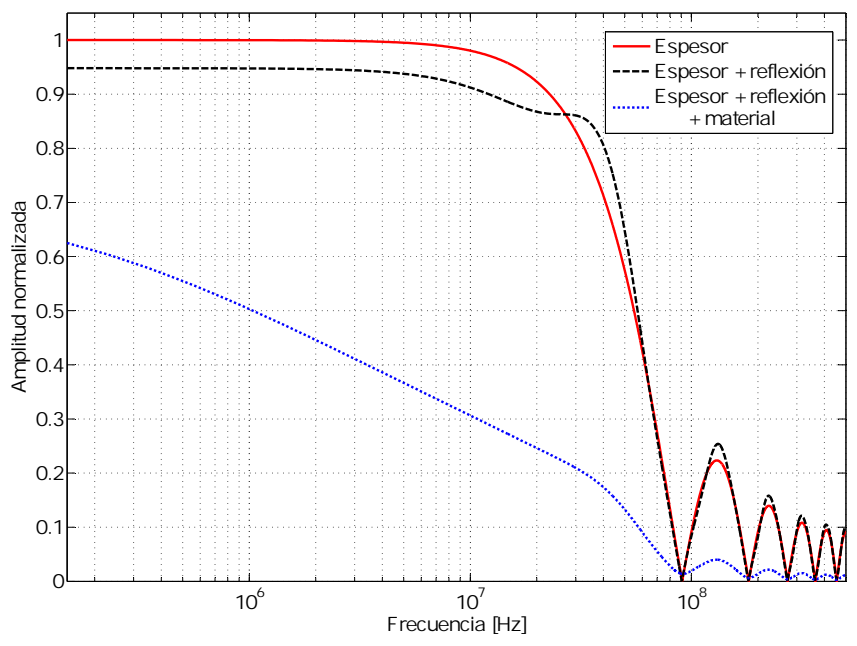

Fig. 5. Módulo normalizado de las transferencias, con $\Gamma_{p}=-0.1$.

En las Figs. 4 y 5 se observan las funciones transferencias de los aspectos físicos del sensor para los dos valores de $\Gamma_{p}$. En línea continua se muestra el efecto de un espesor finito. La curva con trazo a rayas representa tanto el espesor como las reflexiones en las caras posterior y anterior del sensor. Finalmente, la curva de puntos es la transferencia que incluye también las propiedades del material piezoeléctrico.

El espesor del sensor funciona como un filtro pasabajos. Por otro lado, las reflexiones producen grandes cambios en el espectro de la señal respecto del de un sensor ideal. Por consiguiente, es esperable tener imágenes reconstruidas con artefactos, en especial cuando $\Gamma_{p}$ es elevado (ver Fig. 4). En la Fig 5 se puede apreciar el efecto de adaptar las impedancias acústicas entre la cara posterior de la película delgada de PVDF y el substrato donde está apoyada (menor valor de $\Gamma_{p}$ ). Se observa una reducción importante del efecto distorsivo de las reflexiones con respecto al caso desadaptado (Fig. 4). Finalmente, la función transferencia que introduce las propiedades del material, limita la respuesta a frecuencias elevadas $(\geq 1 \mathrm{MHz})$, imponiendo una frecuencia de corte aún menor que en el caso de la función que representa el espesor del film. 


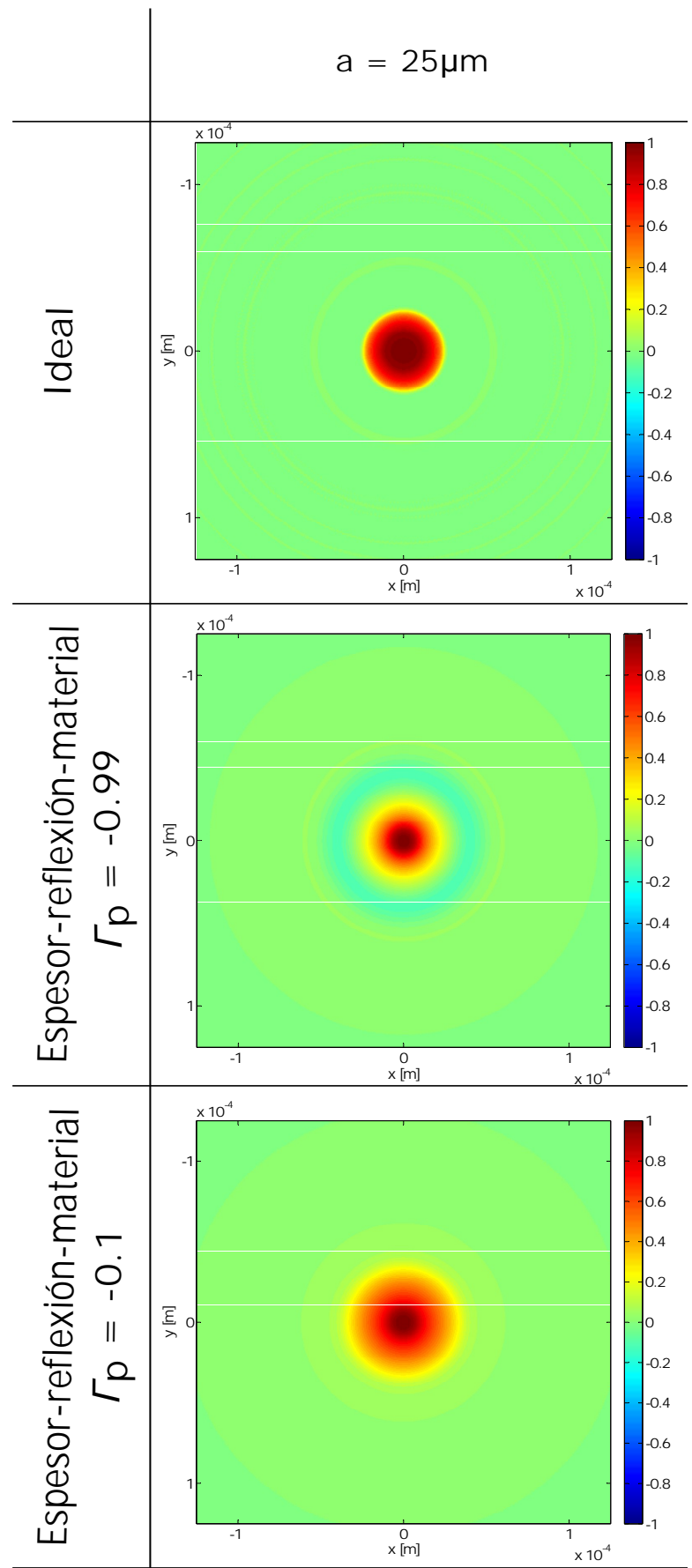

Fig. 6. Reconstrucción 2-D de la distribución inicial de presión inicial $\overline{p_{O}}(\mathbf{r})$, considerando las propiedades física de sensores poliméricos piezoeléctricos de banda ancha con geometría lineal.

En la Fig. 6 se muestra un ejemplo de las reconstrucciones de la distribución inicial de presión $\overline{p_{o}}(\mathbf{r})$, en valores normalizados, para $a=25 \mu \mathrm{m}$. La primera imagen de la figura muestra el caso ideal. Las otras reconstrucciones presentan los efectos producidos al considerar el espesor, el material piezoeléctrico y las reflexiones con $\Gamma_{p}=-0.99$ (imagen central) y $\Gamma_{p}=-0.1$ (imagen inferior). De la figura se puede apreciar que la introducción de los aspectos físicos del sensor afectan notoriamente la reconstrucción de la imagen.

Para facilitar el análisis, en las Figs. 7, 8 y 9 se muestran las reconstrucciones $2-\mathrm{D}$ de $\bar{p}_{o}(x, y=0)$ producidas por esferas con radios $100 \mu \mathrm{m}, 25 \mu \mathrm{m}$ y $10 \mu \mathrm{m}$, respectivamente.

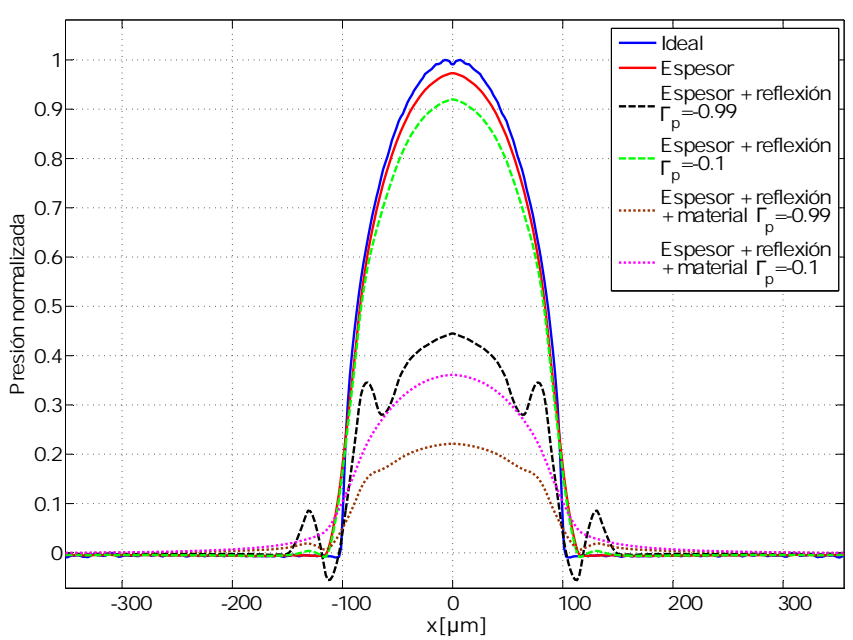

Fig. 7. Reconstrucción 2-D de $\bar{p}_{o}(x, y=0)$ para una fuente de presión esférica de radio $a=100 \mu \mathrm{m}$.

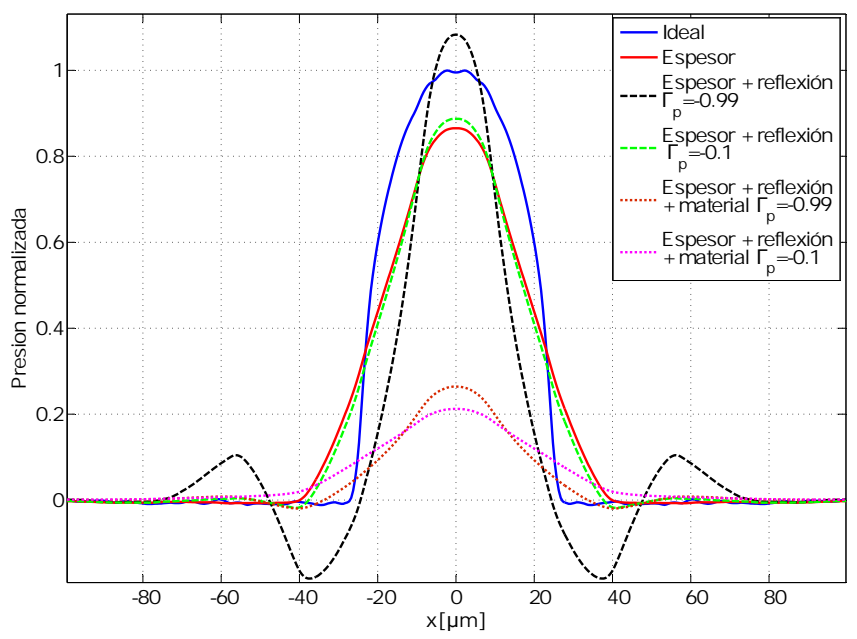

Fig. 8. Reconstrucción 2-D de $\bar{p}_{o}(x, y=0)$ para una fuente de presión esférica de radio $a=25 \mu \mathrm{m}$.

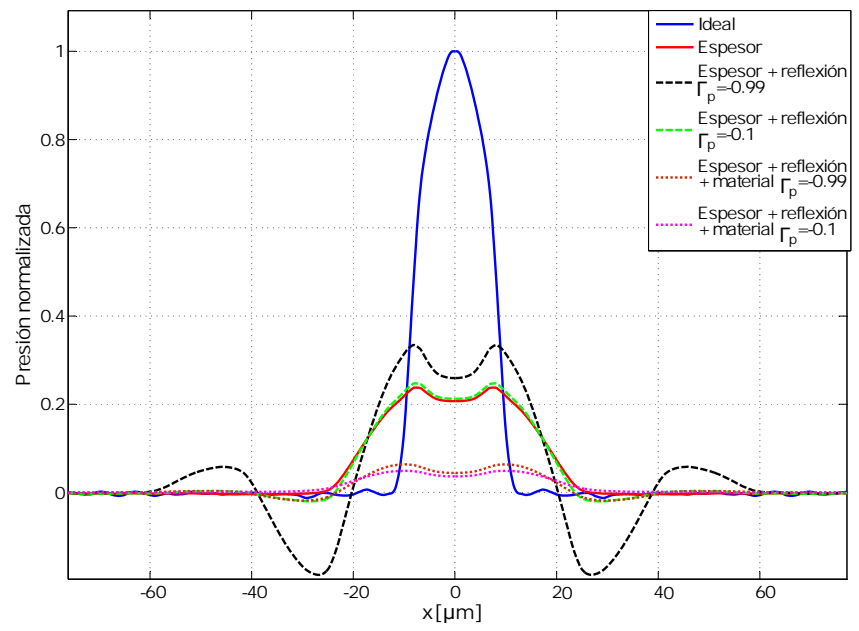

Fig. 9. Reconstrucción 2-D de $\bar{p}_{o}(x, y=0)$ para una fuente de presión esférica de radio $a=10 \mu \mathrm{m}$. 
Para el caso donde se tiene en cuenta un espesor finito (curvas en color rojo), se observa un efecto de filtrado de las componentes de alta frecuencia (pasabajos). En concordancia con esto, a medida que se reduce el tamaño de la fuente de presión, la atenuación y el radio de la esfera reconstruida aumentan en relación al caso ideal.

Cuando se consideran también las reflexiones con $\Gamma_{p}=$ -0.99 (curvas con trazo a rayas color negro) se producen artefactos en la reconstrucción como por ejemplo la aparición de presiones negativas. Esto es debido a que la transferencia de este aspecto físico (ver Fig. 4) posee una importante atenuación a bajas frecuencias $(<1 \mathrm{MHz})$ y una fuerte resonancia acústica en el rango entre $10 \mathrm{MHz}$ y 100 $\mathrm{MHz}$. Es importante destacar que para el caso particular de $a=25 \mu \mathrm{m}$ (Fig. 8), la mayor parte del contenido espectral del pulso de presión coincide con la susodicha resonancia, generando una amplificación en la presión.

Las curvas con trazo a rayas color verde son las mismas simulaciones que para el caso anterior, pero con una mejor adaptación de impedancias, es decir $\Gamma_{p}=-0.1$. Se puede ver una reducción de los efectos adversos de la reflexiones en las distintas interfases entre los materiales que componen al detector.

Las curvas de puntos de colores violeta y marrón representan las reconstrucciones obtenidas al agregar las propiedades del material. La introducción de un factor de atenuación distinto de cero y de la relajación piezoeléctrica reducen las distorsiones provocadas por las reflexiones así como también la amplitud de la señal entregada por el sensor. Además se puede observar que, a pesar de reducir las reflexiones, el radio de las esferas reconstruidas no mejora, siendo mayores que para el caso considerando solo el espesor. Esto denota que la resolución espacial está limitada principalmente por las propiedades del material.

Otra cosa que puede apreciarse observando las Figs. 7, 8 y 9 es que la forma de las curvas también es dependiente del radio de la esfera. Este fenómeno se debe a que a menores valores de $a$, mayor es el ancho de banda de la señal de presión $\mathrm{y}$, por lo tanto, para radios pequeños, se hace más notorio el efecto de atenuación de las componentes de alta frecuencia de la imagen a ser reconstruida.

\section{CONCLUSIONES}

En este trabajo se estudió cómo los aspectos físicos de un sensor no ideal afectan la reconstrucción de imágenes OA. En particular se analizó el caso de sensores poliméricos piezoeléctricos de banda ancha con geometría lineal y su efecto sobre el algoritmo de retroproyección universal.

El estudio se centró sobre tres aspectos físicos del sensor: espesor finito, reflexiones por desadaptación de impedancia acústicas y las propiedades del material polimérico usado para captar las ondas de presión (película delgada de PVDF). Al tener en cuenta el espesor se observó una disminución de la resolución espacial del sistema. El análisis sobre las reflexiones mostró que la elección del sustrato es esencial para lograr imágenes con baja distorsión. Es importante hacer notar que cuando se agregan las propiedades del material, se observa una reducción de los efectos de las reflexiones acústicas en la cara posterior. Esto pone en evidencia que no es necesario lograr una perfecta adaptación de impedancias acústicas para obtener una perfomance satisfactoria, verificando lo mencionado en [10].

En resumen, los resultados obtenidos en este trabajo indican que es necesario caracterizar a priori el sensor que será usado para captar las ondas ultrasónicas en un sistema OA. La información es relevante para determinar si el mismo es o no apto para la aplicación particular. Actualmente nos encontramos trabajando en el desarrollo de un modelo inverso que permita minimizar los efectos adversos introducidos por los aspectos intrínsecos del detector.

\section{AGRADECIMIENTOS}

Este trabajo fue apoyado por los subsidios de la Universidad de Buenos Aires (UBACyT 20020160100052BA) y de la ANPCyT (PICT 2016-2204).

\section{REFERENCIAS}

[1] V. Ntziachristos and D. Razansky. "Molecular imaging by means of multispectral optoacoustic tomography (MSOT)". Chem. Rev., 110:2783-2794, 2010.

[2] V. Ntziachristos, J. Ripoll, L. Wang, and R. Weissleder. "Looking and listening to light: the evolution of whole-body photonic imaging". Nat. Biotechnol., 23:313-320, 2005.

[3] C. Lutzweiler and D. Razansky. "Optoacoustic imaging and tomography: reconstruction approaches and outstanding challenges in image performance and quantification". Sensors, 13:7345-7384, 2013.

[4] M. Xu and L. V. Wang. "Photoacoustic imaging in biomedicine". Rev. Sci. Instrum., 77:041101-1-22, 2006.

[5] A. Rosenthal, V. Ntziachristos, and D. Razansky. "Acoustic inversion in optoacoustic tomograph". Nat. Biotechnol., 9:318-336, 2013.

[6] M. Xu and L. Wang. "Universal back-projection algorithm for photoacoustic computed tomography”. Phys. Rev. E, 71:016706-113, 2005.

[7] L. F. Brown. "Design considerations for piezoelectric polymer ultrasound transducers". IEEE Trans. Ultrason. Ferroelect. Freq. Contr., 47:1377, 2000.

[8] Q. X. Chen and P. A. Payue. "Industrial applications of piezoelectric polymer transducers". Meas. Sci. Technol., 6:249, 1995.

[9] P. Burgholzer, J. Bauer-Marchallinger, H Gruen, M. Haltmeier, and G. Paltauf. "Temporal back-projection algorithms for photoacoustic tomography with integrating line detectors". Inverse Problems, 23:S65-S80, 2007.

[10] A. Fernández Vidal, L. Ciocci Brazzano, C. Matteo, P. Sorichetti, and M. G. González. "Parametric modeling of wideband piezoelectric polymer sensors: design for optoacoustic applications". Rev. Sci. Instrum., 88, 2017.

[11] G. Paltauf, P Hartmair, G Kovachev, and R. Nuster. "Piezoelectric line detector array for photoacoustic tomography". Photoacoustics, $8: 28-36,2017$.

[12] M. Haltmeier and G. Zangerl. "Spatial resolution in photoacoustic tomography: effects of detector size and detector bandwidth". Inverse Problems, 26:125002, 2010.

[13] S. Havriliak and S. Negami. "A complex plane analysis of dispersions in some polymer systems". J.Polym.Sci., Polym. Sym., 14:99, 1966.

[14] M. G. González, P. A. Sorichetti, L. Ciocci Brazzano, and G. D. Santiago. "Electromechanical characterization of piezoelectric polymer thin films in a broad frequency range". Polym. Test., 37:163, 2014. 